\title{
Exacerbation of sleep apnoea by frequent central events in patients with the obstructive sleep apnoea syndrome at altitude: a randomised trial
}

\author{
Yvonne Nussbaumer-Ochsner, ${ }^{1}$ Nicole Schuepfer, ${ }^{1,2}$ Silvia Ulrich, ${ }^{1}$ Konrad E Bloch ${ }^{1}$
}

${ }^{1}$ Pulmonary Division, University Hospital of Zurich, Zurich Center for Integrative Human

Physiology, University of Zurich, Switzerland

${ }^{2}$ Institute of Human Movement Sciences and Sports, Federal Institute of Technology, Zurich, Switzerland

\section{Correspondence to}

Konrad E Bloch, Pulmonary Division, University Hospital of Zurich, Raemistrasse 100, CH-8091 Zurich, Switzerland; konrad.bloch@usz.ch

Received 20 August 2009 Accepted 16 February 2010

\section{ABSTRACT \\ Background Many patients with the obstructive sleep apnoea syndrome (OSA) travel to the mountains for recreational and professional activities while temporarily discontinuing continuous positive airway pressure (CPAP) treatment. A study was undertaken to evaluate the hypothesis that altitude would aggravate their hypoxaemia, sleep-related breathing disturbances and impair daytime performance.}

Methods Thirty-four patients with OSA of median age 62 years (IOR 57-65), median apnoea/hypopnoea index (AHI) 47.5 events/h (IOR 32.4-72.8), residing at $<600 \mathrm{~m}$ were enrolled. A crossover trial randomised for the sequence of altitude exposure was carried out: patients spent 1 day in Zurich $(490 \mathrm{~m})$ and 4 days in the Swiss Alps at $1860 \mathrm{~m}$ and $2590 \mathrm{~m}$ (2 days each) during which continuous positive airway pressure was discontinued. Daily evaluations included polysomnography, symptom questionnaires, physical examination and driving simulator tests.

Results Polysomnography revealed median oxygen saturations at $490 \mathrm{~m}$ and in the first and second nights at 1860 and $2590 \mathrm{~m}$, respectively, of $94 \%, 90 \%, 90 \%$, $86 \%$ and $87 \%$ ( $p<0.01$ between altitudes).

Corresponding median $\mathrm{AHI}$ were 47.5, 85.1, 74.6, 90.0 and 90.9 events/ $h(p<0.01$ between altitudes) with ratios of central to obstructive events of $0.1,0.8,1.0,1.9$ and 1.9 ( $p<0.01$ between altitudes). Tracking performance during simulated driving was significantly impaired at $2590 \mathrm{~m}$ compared with $490 \mathrm{~m}$. Systolic blood pressure and cardiac arrhythmias were increased at altitude.

Conclusions Altitude exposure in untreated patients with OSA aggravates hypoxaemia, increases sleeprelated breathing disturbances due to frequent central apnoeas/hypopnoeas, impairs driving simulator performance and induces cardiovascular stress. These findings have implications for counselling and treating patients with OSA planning to travel to high altitude. ClinicalTrials.gov identifier NCT00514826.

\section{INTRODUCTION}

The obstructive sleep apnoea syndrome (OSA) is caused by repetitive collapse of the upper airway during sleep, provoking apnoeas/hypopnoeas associated with oxygen desaturations and sleep disruption. ${ }^{1}$ Excessive daytime sleepiness, loss of concentration and other symptoms considerably impair the quality of life of patients with OSA, and they are at increased risk of causing traffic accidents $^{2}$ and suffering from cardiovascular disease. ${ }^{3}$ At least $5-10 \%$ of the adult population are affected to some extent. ${ }^{4}$ Nocturnal application of contin- uous positive airway pressure (CPAP) via a mask is currently the most effective treatment. It improves breathing disturbances, sleep quality and daytime symptoms.

Worldwide, a large number of individuals residing at low altitude travel to higher altitudes for work and recreational activities. In various mountain areas including the Alps, the Rocky Mountains and the Andes, settlements are located at altitudes between 1000 and $3000 \mathrm{~m}$, and certain towns, lodges and mountain huts are situated at even higher elevations. High altitude periodic breathing associated with sleep disturbance and altituderelated illness such as acute mountain sickness and high altitude cerebral and pulmonary oedema may affect unacclimatised healthy lowlanders when travelling to high altitudes. ${ }^{5}$ Whether altitude exposure has similar or even more unfavorable consequences in patients with OSA is largely unknown although, because of the high prevalence of the disorder, many are expected to travel to altitude. Based on pathophysiological considerations and uncontrolled observations in a small number of patients, there have been concerns that a stay at altitude might aggravate sleep-related breathing disturbances in patients with OSA and induce central apnoea, ${ }^{67}$ but more robust evidence would be desirable as a basis for counselling and treating patients with OSA travelling to altitude. A randomised controlled trial was performed to evaluate the hypothesis that exposure to moderate altitude would aggravate nocturnal hypoxaemia, sleep and breathing disturbances and impair daytime performance in patients with OSA residing at low altitude and discontinuing CPAP during a 4-day stay at altitude.

\section{METHODS \\ Patients}

Patients with OSA on CPAP therapy were invited to participate in the study by flyers distributed at the University Hospital of Zurich and the Zurich Lung League. Patients aged $>20$ years living at an altitude below $600 \mathrm{~m}$ were considered for participation if a prior diagnosis of OSA was documented by medical records and history confirming excessive sleepiness and an apnoea/hypopnoea index (AHI) $>20 / \mathrm{h}$ before initiation of CPAP treatment. Study inclusion further required $>15$ oxygen desaturations $/ \mathrm{h}$ ( $>3 \%$ dips) during an ambulatory pulse oximetry (PULSOX 300i, Konica Minolta, Osaka, Japan) performed during the last of four nights of CPAP withdrawal that allowed sleep-disordered breathing to return to near the untreated level. ${ }^{89}$ 
Patients also had to have an AHI $>10 / \mathrm{h}$ with predominant obstructive apnoea/hypopnoea during polysomnography at low altitude (Zurich, $490 \mathrm{~m}$ ). The following conditions excluded study admission: unstable cardiovascular disease, any lung disease, internal medical, neurological or psychiatric disease thought to interfere with sleep quality, chronic rhinitis, previous upper airway surgery, use of medication that interferes with ventilation or sleep (acetazolamide, benzodiazepines, opioids).

\section{Protocol}

A randomised controlled crossover trial was performed to evaluate the effects of altitude on untreated OSA. For logistic reasons, during the study period from July to October 2007 a total number of 40 time slots were available that allowed 40 patients to be allocated to two different sequences of interventions and evaluations (A and B) according to a balanced block design. In order to control for a potential order effect, 20 sequence $\mathrm{A}$ (starting with low altitude studies and ending with high altitude studies) and 20 sequence B (starting with high altitude studies and ending with low altitude studies) were available and allocated to time slots at random (figure 1). Details of the sequences and the fact that selecting any particular time slot determined the sequence were concealed from patients until allocation was complete. Randomisation was achieved by letting patients register for one of the available time slots according to their preference (without being aware of the corresponding sequence) until all slots were filled.

Patients discontinued CPAP for a total of eight nights. Days $1-3$ were spent at home $(<600 \mathrm{~m})$ while the effect of CPAP was

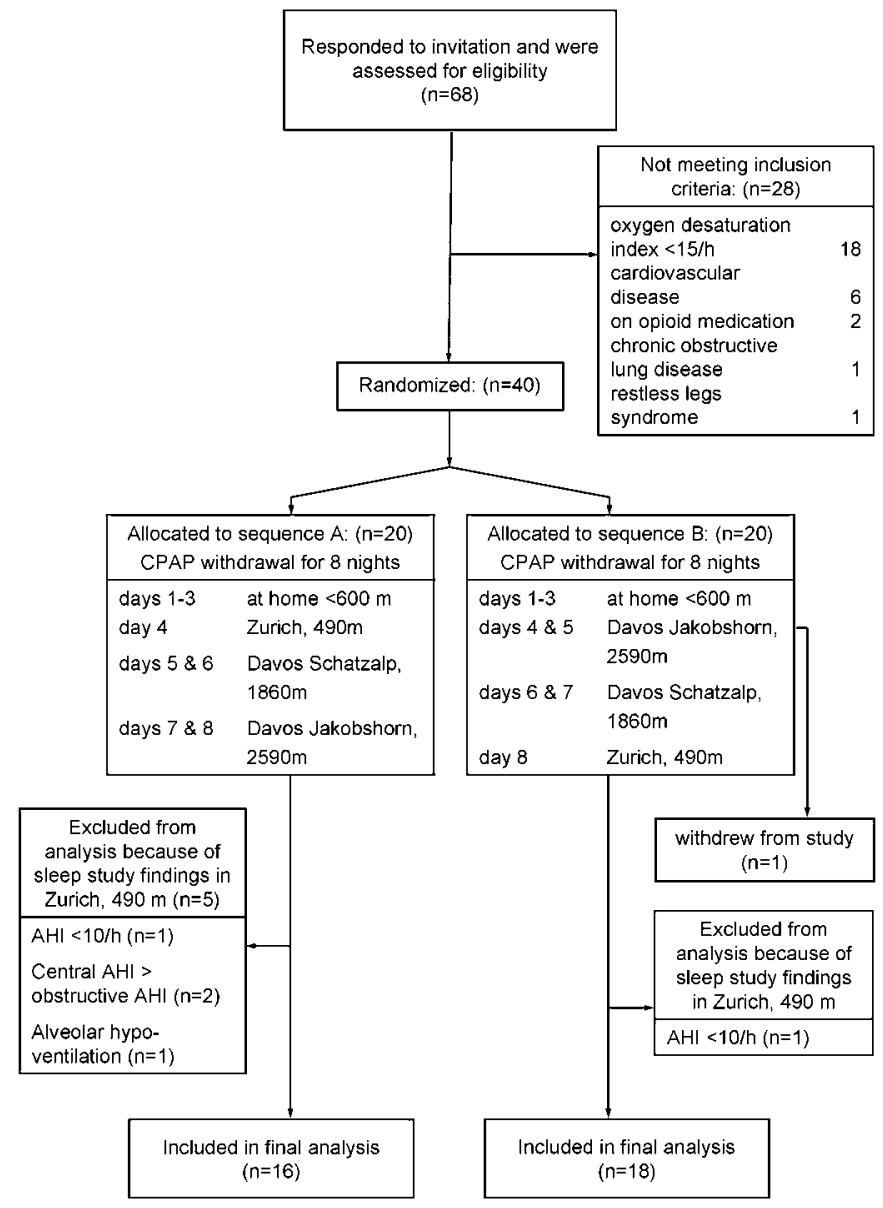

Figure 1 Study flow chart. AHI, apnoea/hypopnoea index; CPAP, continuous positive airway pressure. washed out. Patients assigned to sequence A spent day 4 in Zurich (490 m, $1608 \mathrm{ft}$, barometric pressure (PB) 717 Torr), days 5-6 in Davos Schatzalp (1860 m, $6103 \mathrm{ft}, \mathrm{PB} 607$ Torr) and days 7-8 in Davos Jakobshorn $(2590 \mathrm{~m}, 8498 \mathrm{ft}$, PB 554 Torr). Patients assigned to sequence $B$ spent days $1-3$ at home, days 4-5 in Davos Jakobshorn, days 6-7 in Davos Schatzalp and day 8 in Zurich (figure 1). Transfers between Zurich and Davos, a village in the Swiss Alps, were by a $2.5 \mathrm{~h}$ train ride and between Davos train station and Schatzalp and Jakobshorn, respectively, by cable car. Patients spent nights in sleep laboratories at the study locations.

\section{Outcomes}

Examinations after waking up in the morning included pulse oximetry, body weight and sphygmomanometric blood pressure. Acute mountain sickness was assessed by the Lake Louise protocol $^{10}$ which evaluates headaches, gastrointestinal upset, fatigue/weakness, dizziness/light-headedness, difficulty sleeping and peripheral oedema, tachypnoea, altered mental status and ataxia. The score ranges from 0 to 24 points with increasing severity of acute mountain sickness. Sleepiness was assessed by the Karolinska Sleepiness Scale ${ }^{11}$ ranging from 1 (very awake) to 9 (very tired). During a 30 min test drive with the Divided Attention Driving Simulator, the tracking error (deviation from centre line) and reaction time were recorded. ${ }^{12}$

Polysomnography was performed from approximately 22:00 h to $06: 00 \mathrm{~h}$ according to standard techniques ${ }^{13}$ (Alice5, Respironics AG, Zofingen, Switzerland). Derivations included electroencephalogram (EEG) and electro-oculogram (EOG) leads, submental and bilateral anterior tibial electgromyogram (EMG), pulse oximetry, calibrated respiratory inductive plethysmography, ${ }^{14}$ nasal prong pressure recordings, ${ }^{15}$ bilateral diaphragmatic surface EMG, ${ }^{16}$ end-tidal and transcutaneous carbon dioxide tension $\left(\mathrm{PtccO}_{2}\right) .{ }^{17} 18$ Sleep stages and arousals were scored. ${ }^{19}{ }^{20}$ Apnoea/hypopnoea was defined as a reduction in the inductive plethysmographic sum signal or the nasal pressure swings to $<50 \%$ of the preceding 2 min baseline during $\geq 10$ s. $^{15} 21$ Transient reductions in breathing amplitude to $<50 \%$ baseline for 5-10 s were also scored as apnoeas/hypopnoeas if they occurred as part of a periodic breathing pattern with hyperventilation alternating with central apnoeas/hypopnoeas for at least three consecutive cycles. ${ }^{22}$ Obstructive apnoeas/hypopnoeas were identified by rib cage and abdominal asynchrony and persistent or increasing diaphragmatic EMG activity (figure 2). Central apnoeas/hypopnoeas were identified by absent rib cage-abdominal asynchrony, no signs of inspiratory flow limitation (no flattening of nasal pressure contour) and reduced or absent diaphragmatic EMG activity. The AHI and the oxygen desaturation index ( $>3 \%$ dips) were computed as the number of events per hour of sleep. The sleep study analysts were blinded to the clinical data.

\section{Data analysis}

Data are summarised as medians and interquartile ranges. The effects of altitude and time at any altitude were evaluated by Friedman ANOVA followed by Wilcoxon matched pairs tests if ANOVA indicated a significant overall effect. To test the hypothesis that outcomes at altitude (ie, at $1860 \mathrm{~m}$ and $2590 \mathrm{~m}$, days 1 and 2, respectively) differed from those at $490 \mathrm{~m}$, we planned to perform four comparisons. To account for this, we assumed statistical significance at $\mathrm{p}<0.01$ (ie, we performed a Bonferroni correction by factor 5 rather than 4 to be conservative). In additional exploratory comparisons between data from altitudes $1860 \mathrm{~m}$ and $2590 \mathrm{~m}$, we adjusted the statistical 

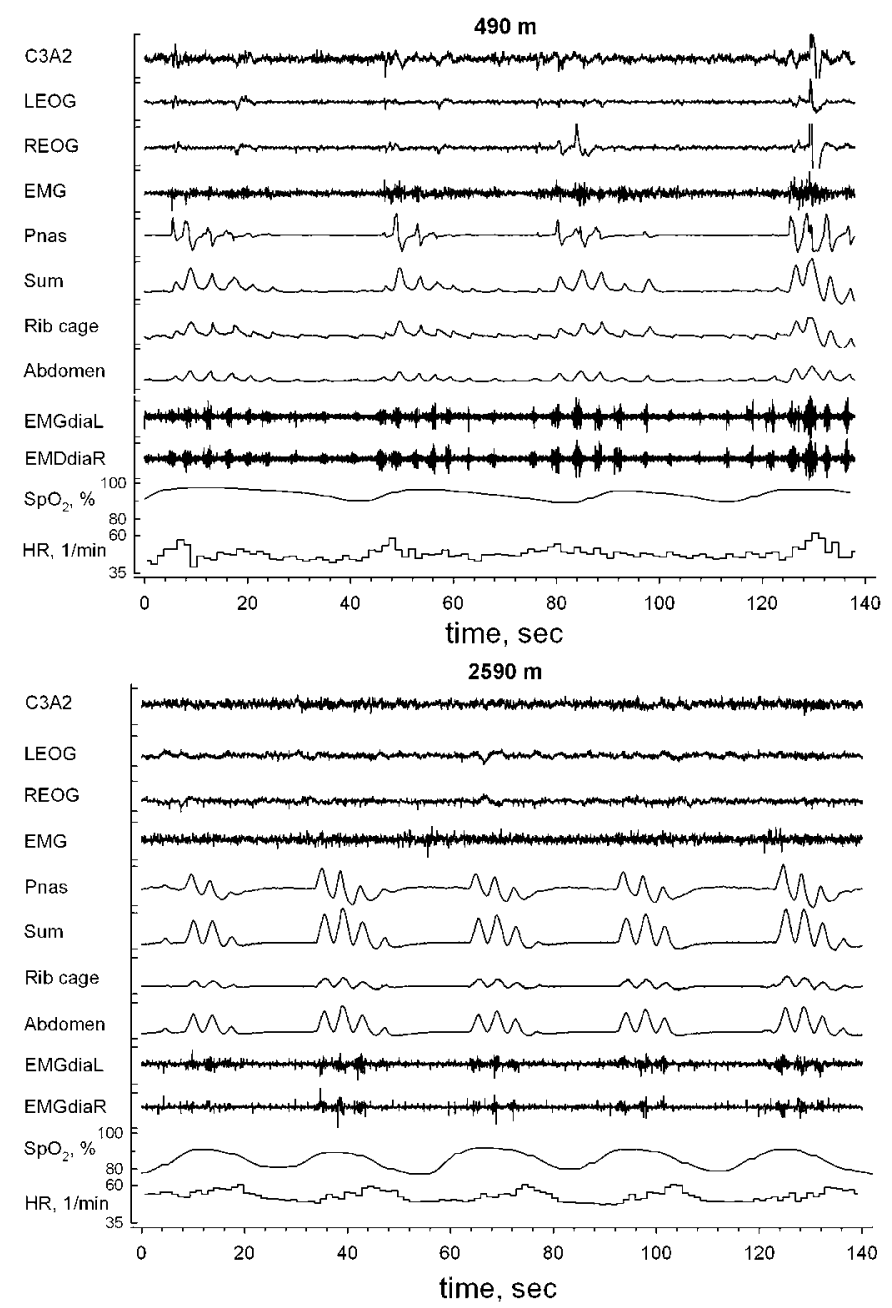

Figure 2 Polysomnographic recordings obtained in a representative patient during NREM sleep stage II at Zurich $(490 \mathrm{~m})$ and at Davos Jakobshorn (2590 m). At $490 \mathrm{~m}$, intermittent absence of nasal pressure swings with paradoxical rib cage and abdominal excursions and persistent diaphragmatic EMG activity during events indicate that apnoeas are obstructive. At $2590 \mathrm{~m}$, the intermittent absence of rib cage abdominal motion and diaphragmatic EMG activity indicates that apnoeas are central. Traces are a central EEG derivation (C3A2), left and right EOG (LEOG, REOG), submental EMG, nasal pressure swings (Pnas), inductive plethysmographic sum, rib cage and abdominal volume curves, left and right diaphragmatic EMG (EMGdiaL, EMGdiaR), oxygen saturation by pulse oximetry $\left(\mathrm{Spo}_{2}\right)$ and heart rate $(\mathrm{HR})$.

significance of the $\mathrm{p}$ value from 0.05 by a factor of 10 to 0.005 (10 possible comparisons between data from all three altitudes and days 1 and 2). The primary outcomes were oxygen saturation and AHI, and secondary outcomes were sleep quality and vigilance, symptoms and physical findings. Clinically relevant differences were assumed to be $2 \%$ (SD 2\%) for oxygen saturation and $10 / \mathrm{h}$ (SD 15/h) for the AHI. Power calculation indicated a minimal sample size of 29 to detect clinically relevant differences in primary outcomes with a power of $80 \%(\alpha=0.01)$. To account for potential drop-outs, our aim was to recruit 40 patients.

\section{RESULTS}

\section{Patients}

Figure 1 illustrates the patient flow. Of 68 patients who applied to participate in the study, 40 met the inclusion criteria and were randomised. One patient withdrew after one night at Jakobshorn because of lack of motivation. Data from five patients had to be excluded according to predefined criteria because baseline polysomnography at low altitude (Zurich) revealed an obstructive AHI $<10 / h$ in two, predominant central apnoeas/hypopnoeas in two and alveolar hypoventilation in one. Data from 34 patients (32 men, 2 women) were available for analysis. Median (IOR) age was 62 (57-65) years and body mass index was 33.7 $(27.3-38.1) \mathrm{kg} / \mathrm{m}^{2}$. Patients had been on CPAP treatment for 41 (20-56) months. Comorbidities included arterial hypertension in $68 \%$ and diabetes mellitus in $15 \%$ of subjects; $38 \%$ were receiving ACE inhibitors/angiotensin II antagonists, 35\% diuretics, $24 \% \beta$ blockers and $18 \%$ calcium channel blockers. No serious adverse events requiring medical treatment occurred.

\section{Sleep studies}

The sleep study results are summarised in table 1 and data on nocturnal ventilation, oxygen saturation and heart rate are shown in table 2. A representative recording is shown in figure 2. A comparison of the data from subjects undergoing sequences $A$ and $B$ did not reveal significant differences in oxygen saturation, AHI, percentage slow wave sleep and sleep efficiency, so the data from the two groups are presented together. Compared with values recorded at $490 \mathrm{~m}$, oxygen saturation was significantly reduced at $1860 \mathrm{~m}$ and even more so at $2590 \mathrm{~m}$, particularly during REM sleep. Transcutaneous $\mathrm{PCO}_{2}$ was highest at $490 \mathrm{~m}$ during REM sleep and lowest at $2590 \mathrm{~m}$ during NREM sleep. Studies at $490 \mathrm{~m}$ revealed severe OSA and only occasional central apnoeas/hypopnoeas according to inclusion criteria. On the first and second day at 1860 and $2590 \mathrm{~m}$, respectively, the AHI increased significantly to medians of $147 \%, 141 \%$ and $166 \%, 167 \%$ of the $490 \mathrm{~m}$ baseline value. This was related to the emergence of frequent central apnoeas/hypopnoeas during NREM sleep while obstructive apnoeas/hypopnoeas persisted (figure 3). Sleep quality worsened significantly at altitude as reflected in an increased arousal index, reduced sleep efficiency and slow wave sleep (NREM stages III and IV) at $2590 \mathrm{~m}$. The number of arousals was significantly correlated with the AHI at all altitudes (at $490 \mathrm{~m}$ : Spearman $\mathrm{R}=0.77 ; 1860 \mathrm{~m}$, day 1: $\mathrm{R}=0.70 ; 1860 \mathrm{~m}$, day $2: \mathrm{R}=0.77 ; 2590 \mathrm{~m}$, day $1: \mathrm{R}=0.68 ; 2590 \mathrm{~m}$, day 2: $\mathrm{R}=0.77 ; \mathrm{p}<0.01$ in all instances). Compared with $490 \mathrm{~m}$, the heart rate and the number of premature beats were significantly increased at altitude.

Comparisons of sleep studies on the first versus the second night at corresponding altitude revealed no effect of acclimatisation on oxygen saturation and transcutaneous $\mathrm{PCO}_{2}$.

\section{Symptoms and daytime findings}

In the morning the Karolinski Sleepiness Scale indicated that patients felt moderately sleepy (table 3). They reported moderate symptoms of acute mountain sickness at altitude but the Lake Louise score was also increased at $490 \mathrm{~m}$. None of the patients had symptoms or signs of high altitude pulmonary oedema. There was an increase in weight at $2590 \mathrm{~m}$ compared with 490 and $1860 \mathrm{~m}$, and this was associated with ankle oedema. Systolic blood pressure was increased at $1860 \mathrm{~m}$ and $2590 \mathrm{~m}$ above values at $490 \mathrm{~m}$ (table 3). In two patients antihypertensive medication needed to be adapted at $2590 \mathrm{~m}$ because of an excessive rise in blood pressure. Driving simulator tests revealed significantly increased tracking errors at $2590 \mathrm{~m}$ compared with the lower altitudes (figure 4).

\section{DISCUSSION}

We performed a randomised controlled crossover trial in patients with moderate to severe OSA during temporary CPAP discontinuation to evaluate the effects of a 4-day stay at moderate altitudes 
Table 1 Sleep studies $(n=32)$

\begin{tabular}{|c|c|c|c|c|c|}
\hline & \multirow{2}{*}{$\begin{array}{l}\text { Zurich } 490 \mathrm{~m} \\
\text { 1st day }\end{array}$} & \multicolumn{2}{|c|}{ Davos Schatzalp $1860 \mathrm{~m}$} & \multicolumn{2}{|c|}{ Davos Jakobshorn $2590 \mathrm{~m}$} \\
\hline & & 1st day & 2nd day & 1st day & 2nd day \\
\hline Total sleep time (min) & $369(345-388)$ & $423 *(389-467)$ & $427^{*}(405-458)$ & $391 \neq(303-457)$ & $401 † \ddagger(300-423)$ \\
\hline Sleep efficiency (\%) & $86(80-89)$ & $82(76-87)$ & $79 *(72-88)$ & $78^{*}(61-84)$ & $71 * \dagger ‡(61-84)$ \\
\hline NREM $3+4(\%)$ & $14(8-22)$ & $12(4-19)$ & $14(8-17)$ & $6^{*} \dagger \neq(0-12)$ & $9^{*} \neq(2-16)$ \\
\hline REM (\%) & $13(9-18)$ & $13(10-18)$ & $13(9-20)$ & $10 \dagger \neq(4-16)$ & $10(7-15)$ \\
\hline Arousal index $(1 / h)$ & $35.5(25.1-43.2)$ & $41.8^{*}(33.5-61.1)$ & $45.5^{*}(29.9-56.7)$ & $51.3^{*}(37.6-65.5)$ & $49.3^{*}(34.4-65.7)$ \\
\hline
\end{tabular}

Values are median (IQR)

${ }^{*} \mathrm{p}<0.01$ vs $490 \mathrm{~m}$.

$\mathrm{tp}<0.005$ vs $1860 \mathrm{~m}$, day 1 .

$\neq \mathrm{p}<0.005$ vs $1860 \mathrm{~m}$, day 2 .

(1860 $\mathrm{m}$ and $2590 \mathrm{~m}$ ) on sleep, breathing and daytime performance. The main findings were a progressive reduction in nocturnal oxygen saturation associated with hyperventilation and a major increase in the AHI, particularly at the highest altitude, due to frequent central apnoeas/hypopnoeas while obstructive events persisted. Correspondingly, sleep quality and driving simulator performance were impaired at altitude. Further consequences of altitude exposure were an increased prevalence of nocturnal cardiac arrhythmias, a significant rise in heart rate and in systolic blood pressure and a weight gain with peripheral oedema. We conclude that a stay at moderate altitude in untreated patients with OSA is associated with unfavorable health effects.

In healthy subjects, hypobaric hypoxia at altitudes $>2000 \mathrm{~m}$ induces periodic breathing with central apnoea/hypopnoea. ${ }^{23-25}$ Breathing instability is related to hypoxic stimulation of ventilation resulting in a reduced $\mathrm{CO}_{2}$ reserve-that is, an eupnoeic $\mathrm{PCO}_{2}$ close to the apnoea threshold promoting apnoea at a minor rise in ventilation because enhanced chemosensitivity causes a ventilatory overshoot. ${ }^{26}$ Data on breathing patterns and sleep in patients with OSA at altitude are scant. During exposure to a simulated altitude of $2750 \mathrm{~m}\left(\mathrm{FIO}_{2} 16.05 \%\right.$ at $\left.610 \mathrm{~m}, \mathrm{~PB} 700 \mathrm{~mm} \mathrm{Hg}\right)$, five patients with OSA had an increase in central apnoeas and a reduction in obstructive apnoeas compared with studies in normoxia at sea level. ${ }^{6}$ In 11 patients with OSA undergoing repeated sleep studies at an altitude $>2400 \mathrm{~m}$, at $1370 \mathrm{~m}$ and at sea level (five patients), the AHI decreased with descent in altitude due to a reduction in central apnoeas. ${ }^{7}$ Our randomised trial corrobo- rates and extends these observational studies by confirming a dose-response relationship between progressive hypoxia at increasing altitude, decreasing transcutaneous $\mathrm{PCO}_{2}$ reflecting more pronounced hyperventilation and emergence of frequent central apnoeas/hypopnoeas. The number of events scored as central might have been even higher if we had not applied stringent criteria requiring absence of signs of inspiratory flow limitation, perfect synchronisation of rib cage-abdominal motion recorded by calibrated inductive plethysmography and a reduced or absent diaphragmatic surface EMG activity. This conservative definition excluded central and mixed events with partial upper airway occlusion. The increase in central apnoea index at altitude occurred predominantly during NREM sleep but rarely during REM sleep (figure 3). This was consistent with a greater increase in hypoxic and hypercapnic ventilatory drive in NREM sleep compared with REM sleep at altitude ${ }^{25}$ and a widened $\mathrm{CO}_{2}$ reserve and/or absence of a post-hyperventilation-induced apnoeic threshold in REM sleep. ${ }^{27}$

In our study there was no significant change in the obstructive AHI at altitude (table 2). Presumably, factors contributing to upper airway patency during hypoxia such as chemical stimulation of dilator muscles were offset by factors promoting airway collapse such as stimulation of ventilatory pump muscles that increase the upper airway transmural pressure gradient during vigorous inspiratory efforts. ${ }^{28} 29$ A shorter duration of obstructive apnoea/hypopnoea at altitude (table 2) is consistent with a lower arousal threshold and/or a lower recruitment

Table 2 Nocturnal ventilation, oxygen saturation and heart rate $(n=34)$

\begin{tabular}{|c|c|c|c|c|c|}
\hline & \multirow{2}{*}{$\begin{array}{l}\text { Zurich } 490 \mathrm{~m} \\
1^{\text {st }} \text { day }\end{array}$} & \multicolumn{2}{|c|}{ Davos Schatzalp 1860 m } & \multicolumn{2}{|c|}{ Davos Jakobshorn 2590 m } \\
\hline & & $1^{\text {st }}$ day & $2^{\text {nd }}$ day & $1^{\text {st }}$ day & $2^{\text {nd }}$ day \\
\hline $\mathrm{SpO}_{2}$, entire night $(\%)$ & $94(93-95)$ & $90 *(88-91)$ & $90 *(88-91)$ & $86^{*} \dagger \ddagger(84-89)$ & $87^{*} \dagger \ddagger(84-89)$ \\
\hline $\mathrm{SpO}_{2}$ during REM (\%) & $94 \S(93-95)$ & $89 * \S(88-91)$ & $90 * \S(88-91)$ & $85^{*} \dagger \neq \S(83-89)$ & $86^{*} \dagger \ddagger \S(84-89)$ \\
\hline $\mathrm{SpO}_{2}$ during REM (\%) & $94(90-95)$ & $87^{*}(84-91)$ & $88 *(85-91)$ & $83^{*} † \ddagger(78-88)$ & $84^{*} † \ddagger(81-87)$ \\
\hline $\mathrm{PtccO}_{2}$, entire night $(\mathrm{mm} \mathrm{Hg})$ & $49(44-50)$ & $39 *(36-41)$ & $39 *(37-41)$ & $37^{*}(33-42)$ & $37^{*} \dagger \neq(33-39)$ \\
\hline $\mathrm{PtcCO}_{2}$ during NREM (mm Hg) & $49(46-50)$ & $40 * \S(37-44)$ & $39 * \S(36-42)$ & $38^{*}(35-42)$ & $39 *(34-40)$ \\
\hline $\mathrm{PtcCO}_{2}$ during REM (mm Hg) & $51(48-51)$ & $41^{*}(37-44)$ & $43^{*}(37-44)$ & $40^{*}(36-42)$ & $39 *(34-42)$ \\
\hline Oxygen desaturation index (1/h) & $37.3(14.6-52.7)$ & $67.6^{*}(37.6-88.2)$ & $57.4^{*}(32.1-86.1)$ & $80.6^{*} \dagger \neq(52.4-103.4)$ & $71.5^{*} \dagger \neq(43.4-98.6)$ \\
\hline Total AHI (events/h) & $51.2(31.7-74.4)$ & $85.1^{*}(53.9-94.3)$ & $68.5^{*}(51.3-95.7)$ & $90.0^{*} \dagger \neq(64.2-103.2)$ & $88.6^{*} \ddagger(62.4-108.4)$ \\
\hline Obstructive AHI (events/h) & $39.4(24.1-65.8)$ & $32.1(21.3-71.8)$ & $33.0(15.3-60.0)$ & $32.0(3.3-54.6)$ & $25.2(6.5-58.8)$ \\
\hline Central AHI (events/h) & $2.4(0.4-8.8)$ & $25.4^{*}(17.0-55.9)$ & $32.7^{*}(14.2-43.7)$ & $51.3^{*} \dagger \ddagger(32.8-75.5)$ & $49.4^{*} \ddagger(22.6-57.8)$ \\
\hline Obstructive apnoea duration (s) & $21.0(16.4-24.9)$ & $22.8(17.7-26.6)$ & $22.6(18.5-27.8)$ & $18.8 \neq(13.3-22.8)$ & $18.5 \neq(15.4-24.1)$ \\
\hline Central apnoea duration (s) & $11.6(8.9-18.2)$ & $15.5^{*}(13.2-19.3)$ & $15.1(12.7-19.4)$ & $14.2 \dagger(12.5-16.7)$ & $15.3(12.2-18.3)$ \\
\hline Heart rate (1/min) & $57.2(54.7-61.8)$ & $61.1 *(57.2-66.4)$ & $61.5^{*}(57.4-69.2)$ & $66.5^{*}(58.4-72.0)$ & $62.6^{*}(57.7-68.8)$ \\
\hline Premature beats, entire night $(1 / h)$ & $3.2(0.6-11.2)$ & $6.7(1.2-33.6)$ & $9.8^{*}(3.0-31.0)$ & $7.2(2.2-29.6)$ & $6.1(1.2-30.4)$ \\
\hline
\end{tabular}

\footnotetext{
Values are median (IOR)

${ }^{*} \mathrm{p}<0.01$ vs $490 \mathrm{~m}$.

$\mathrm{tp}<0.005$ vs $1860 \mathrm{~m}$, day 1 .

$\neq \mathrm{p}<0.005$ vs $1860 \mathrm{~m}$, day 2 .

$\S \mathrm{p}<0.005$ vs REM.

$\mathrm{AHI}$, apnoea/hypopnoea index; $\mathrm{PtcCO}_{2}$, transcutaneous carbon dioxide tension; $\mathrm{Spo}_{2}$, oxygen saturation.
} 
Apnoea/Hypopnoea Index, NREM Sleep

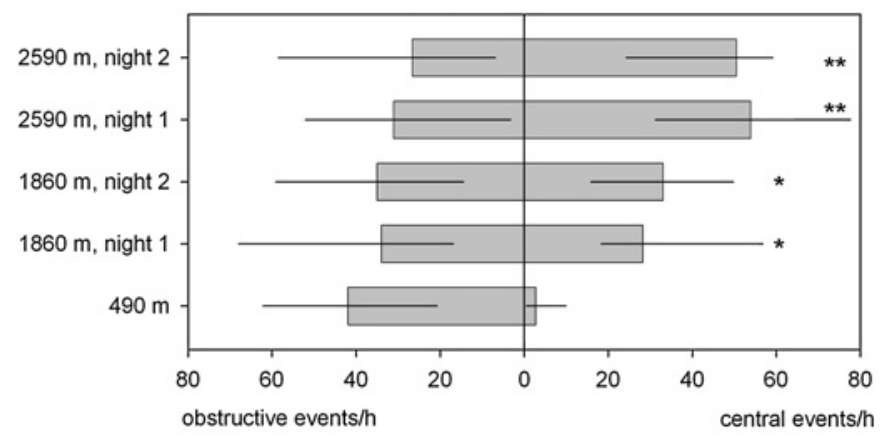

Apnoea/Hypopnoea Index, REM Sleep

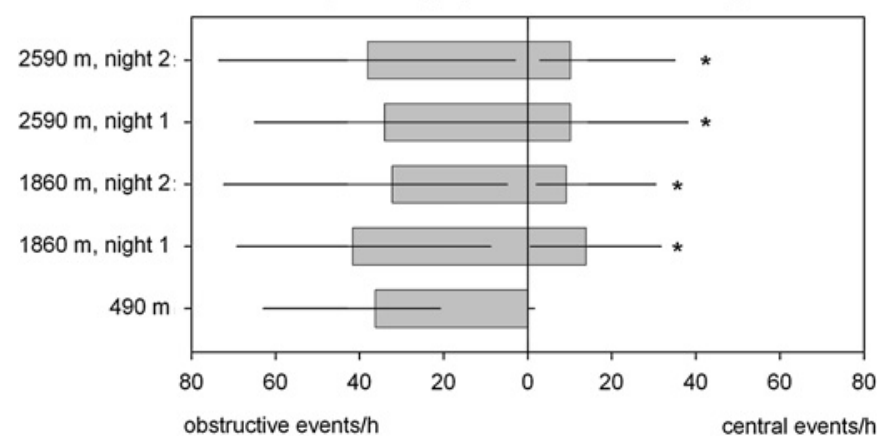

Figure 3 Sleep-related breathing disturbances at $490 \mathrm{~m}$ and in the first and second night at $1680 \mathrm{~m}$ and $2590 \mathrm{~m}$, respectively. The bars and horizontal lines represent median values and quartile ranges of obstructive and central apnoea/hypopnoea indices at the different locations. The upper panel represents events during NREM sleep and the lower panel corresponding values during REM sleep. At higher altitudes, the total apnoea/hypopnoea index increased significantly, related to the emergence of central apnoea/hypopnoea during NREM sleep. ${ }^{*} \mathrm{p}<0.01$ vs $490 \mathrm{~m} ;{ }^{* *} \mathrm{p}<0.01$ vs $490 \mathrm{~m}$ and vs $1860 \mathrm{~m}$.

threshold of upper airway dilator muscles in hypoxia compared with normoxia. ${ }^{29}$

Sleep fragmentation and a reduced amount of slow wave sleep have been reported previously in healthy subjects at altitude. ${ }^{30-32}$ Correspondingly, we observed that sleep quality in patients with OSA was impaired at altitude, as reflected in a reduction in sleep efficiency and slow wave sleep and frequent arousals (table 1). Correlation analysis indicated that $46-59 \%$ of the variation in the arousal index was explained by the AHI, suggesting a significant contribution of breathing disturbances to sleep fragmentation although other factors might additionally have impaired sleep quality in hypoxia. ${ }^{32}$ In five patients with OSA studied at a simulated altitude of $2750 \mathrm{~m}$ (isobaric hypoxia), ${ }^{6}$ no significant changes in sleep stages compared with sea level studies were observed, possibly related to the small sample size.

Questionnaire evaluation indicated that patients perceived moderate headache, poor sleep quality and sleepiness even at low altitude (table 3), which was probably due to CPAP withdrawal. There was no clear further increase in these symptoms at altitude (table 3 ). We cannot exclude the possibility that the positive attitude towards a stay at altitude may have influenced the perception of altitude-related symptoms in the study participants. However, objective testing during a simulated car drive indicated reduced psychomotor vigilance at $2590 \mathrm{~m}$ (table 3), highlighting the potential clinical relevance of exposure to hypoxia in patients with OSA who already have cognitive dysfunction in normoxia. ${ }^{12}$

OSA is associated with cardiac arrhythmia and systemic hypertension at low altitude. ${ }^{3}$ In this study, patients had a higher prevalence of nocturnal premature beats and a rise in heart rate and systolic blood pressure at altitude (tables 2 and 3), suggesting an additional cardiovascular effect of sustained hypoxia and of the more pronounced breathing disturbances at altitude, possibly related to sympathetic activation and parasympathetic withdrawal. ${ }^{33} 34$ The weight gain we observed in association with the development of peripheral oedema (table 3) is consistent with water retention, possibly mediated by sympathetic overstimulation, rise in systemic and pulmonary artery pressure and activation of the renin-angiotensin-aldosterone system in patients with OSA with symptoms of acute mountain sickness. ${ }^{35}$

In conclusion, this randomised crossover trial in patients with OSA indicates that hypoxia, breathing and sleep disturbances are more severe at altitude and are associated with signs of cardiovascular stress and impaired driving simulator performance. The results caution against altitude exposure of untreated patients with OSA, and may help in the design of

Table 3 Daytime evaluation

\begin{tabular}{|c|c|c|c|c|c|}
\hline & \multirow{2}{*}{ Zurich 490 m } & \multicolumn{2}{|c|}{ Davos Schatzalp 1860 m } & \multicolumn{2}{|c|}{ Davos Jakobshorn 2590 m } \\
\hline & & $1^{\text {st }}$ day & $2^{\text {nd }}$ day & $1^{\text {st }}$ day & $2^{\text {nd }}$ day \\
\hline $\mathrm{SpO}_{2}(\%)$ & $95(94-96)$ & $93^{*}(92-94)$ & $94^{*}(93-94)$ & $91^{*}(90-93)$ & $92^{*}(91-93)$ \\
\hline Weight (kg) & $98.8(82.7-110.6)$ & $98.0 *(83.5-109)$ & $98.5^{*}(84-108)$ & $99.0^{*} \dagger \neq(85.0-112.0)$ & $100.0^{*} \dagger \neq(84-110)$ \\
\hline Heart rate $(1 / \mathrm{min})$ & $61(57-66)$ & $72^{*}(66-77)$ & $73^{*}(69-77)$ & $74^{*}(64-80)$ & $74^{*}(64-78)$ \\
\hline Systolic blood pressure $(\mathrm{mm} \mathrm{Hg})$ & $130(120-140)$ & $143^{*}(135-156)$ & $145^{*}(132-152)$ & $144^{*}(132-160)$ & $144^{*}(130-162)$ \\
\hline Diastolic blood pressure $(\mathrm{mm} \mathrm{Hg})$ & $85(80-90)$ & $86(80-92)$ & $86(80-95)$ & $85(81-90)$ & $90(81-97)$ \\
\hline Peripheral oedema§ & $0(0-0)$ & $0 *(0-2)$ & $0 *(2-2)$ & $0^{*}(0-2)$ & $0 *(0-2)$ \\
\hline Driving simulator reaction time (s) & $3.4(2.3-10.0)$ & $3.1(2.5-10.0)$ & $2.9(2.3-4.3)$ & $10.0(2.7-10.0)$ & $10.0 \neq(3.0-10.0)$ \\
\hline Driving simulator tracking error & $0.38(0.32-0.55)$ & $0.42(0.34-0.55)$ & $0.40(0.30-0.47)$ & $0.70^{*} \dagger \neq(0.51-0.90)$ & $0.67^{*} \dagger \neq(0.49-0.89)$ \\
\hline $\begin{array}{l}\text { Lake Louise acute mountain sickness } \\
\text { score }\end{array}$ & $2.5(2.0-4.0)$ & $3.0(2.0-4.0)$ & $2.0(1.0-3.0)$ & $4.0 \neq(3.0-5.0)$ & $3.0 \neq(2.0-5.0)$ \\
\hline Difficulty sleeping§ & $1(1-2)$ & $1(1-1)$ & $1+(0-1)$ & $1.5 \neq(1-2)$ & $1(1-2)$ \\
\hline Karolinksa sleepiness score & $4(3-6)$ & $3^{*}(3-4)$ & $3^{*}(2-3)$ & $3(3-4)$ & $3(2-3)$ \\
\hline
\end{tabular}

Data were obtained in the morning after sleep studies. Values are median (IQR).

${ }^{*} \mathrm{p}<0.01$ vs $490 \mathrm{~m}$.

$\dagger \mathrm{p}<0.005$ vs $1860 \mathrm{~m}$, day 1 .

$\neq \mathrm{p}<0.005$ vs $1860 \mathrm{~m}$, day 2 .

§Peripheral oedema was rated as part of the Lake Louise acute mountain sickness protocol with 0 (absent), 1 (at one location) or 2 points (at two or more locations). Difficulty sleeping was also rated within the Lake Louise protocol with 0 (slept as usual) to 3 points (could not sleep at all).

$\mathrm{SpO}_{2}$, oxygen saturation. 
Figure 4 Performance during a divided attention driving simulator test in a patient with obstructive sleep apnoea at $490 \mathrm{~m}$ and at $2590 \mathrm{~m}$. During the 30 min test drive the patient had to stay in the centre of the road displayed on a computer screen by using a steering wheel. At the same time he had to check numbers appearing in the four corners of the screen. As soon as the number 2 appeared he had to press a button. The computer recorded the distance from the road centre line and the elapsed time until the number 2 was confirmed by pressing the button. In the upper panel the plot shows results obtained at $490 \mathrm{~m}$. The average response time to the number 2 was $2.6 \mathrm{~s}$ (each response is shown as a circle in the upper panel). The patient was able to stay near the centre line (lower panel). In contrast, at $2590 \mathrm{~m}$, the patient missed the number 2 several times (circles at the upper limit of the panel labelled reaction time) and he had great difficulties staying on the road. In the lowest panel, his driving path shows large fluctuations, frequently crossing the lateral boundaries of the road (two horizontal lines). Deviation from the center line is expressed in arbitrary units (a.u.).

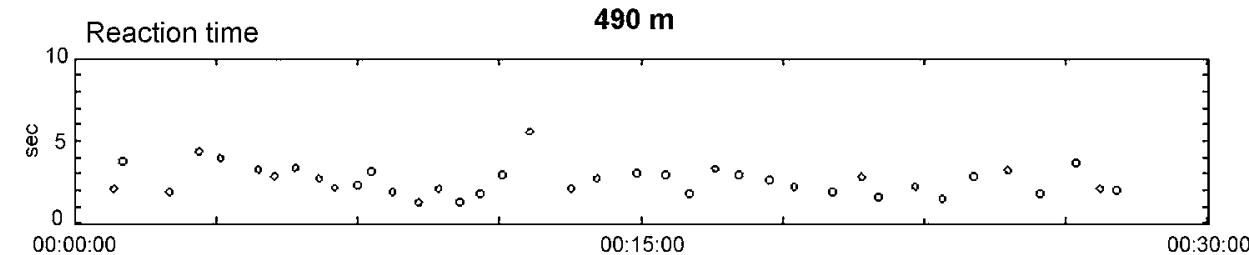

Deviation from road
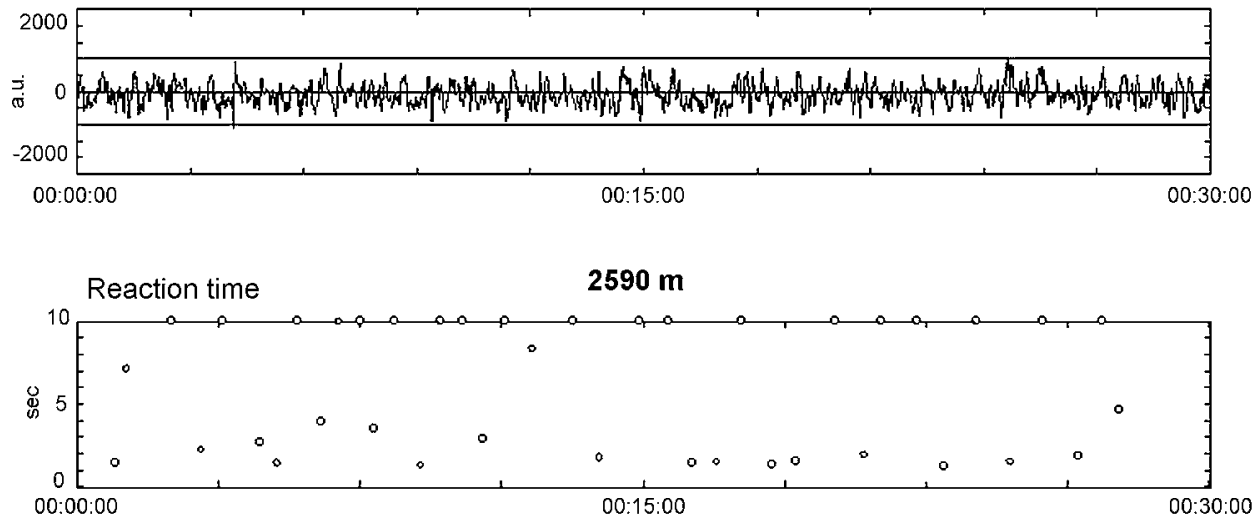

Deviation from road

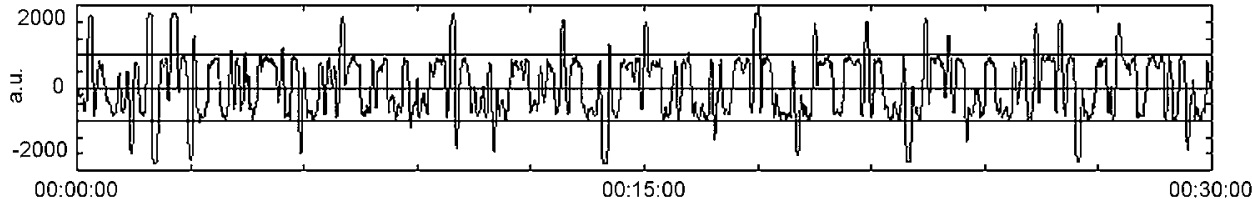

further studies evaluating the effectiveness of CPAP and other treatment modalities that might improve subjective well-being and reduce the potential risk of traffic accidents and cardiovascular complications in patients with OSA at altitude.

Funding Swiss National Science Foundation, Lung Leagues of Zurich and of Schaffhausen, Switzerland.

Competing interests None.

Ethics approval This study was conducted with the approval of the ethics committee of the University Hospital of Zurich.

Contributors All listed authors have contributed to the conception and design, acquisition, analysis and interpretation of data, drafting the article or revising it critically for important intellectual content. They have provided final approval of the version submitted.

Provenance and peer review Not commissioned; externally peer reviewed.

\section{REFERENCES}

1. Stradling JR, Davies RJ. Sleep. 1: obstructive sleep apnoea/hypopnoea syndrome: definitions, epidemiology, and natural history. Thorax 2004:59:73-8.

2. Teran-Santos J, Jimenez-Gomez A, Cordero-Guevara J. The association between sleep apnea and the risk of traffic accidents. Cooperative Group Burgos-Santander. N Engl J Med 1999;340:847-51.

3. Bradley TD, Floras JS. Obstructive sleep apnoea and its cardiovascular consequences. Lancet 2009;373:82-93.

4. Young T, Peppard PE, Gottlieb DJ. Epidemiology of obstructive sleep apnea: a population health perspective. Am J Respir Crit Care Med 2002:165:1217-39.

5. Hackett PH, Roach RC. High-altitude illness. N Engl J Med 2001;345:107-14.

6. Burgess KR, Cooper J, Rice A, et al. Effect of simulated altitude during sleep on moderate-severity OSA. Respirology 2006:11:62-9.

7. Patz D, Spoon M, Corbin R, et al. The effect of altitude descent on obstructive sleep apnea. Chest 2006;130:1744-50.

8. Fiz JA, Abad J, Ruiz J, et al. nCPAP treatment interruption in OSA patients. Respir Med 1998;92:28-31

9. Yang 0, Phillips CL, Melehan KL, et al. Effects of short-term CPAP withdrawal on neurobehavioral performance in patients with obstructive sleep apnea. Sleep 2006;29:545-52
10. Sutton JR, Coates G, Huston CS, eds. The Lake Louise acute mountain sickness scoring system. Hypoxia and molecular medicine: Proceedings of the 8th International Hypoxia Symposium. Burlington, Canada: Queen City Printers, 1993.

11. Kaida K, Takahashi M, Akerstedt T, et al. Validation of the Karolinska sleepiness scale against performance and EEG variables. Clin Neurophysiol 2006;117:1574-81.

12. Juniper M, Hack MA, George CF, et al. Steering simulation performance in patients with obstructive sleep apnoea and matched control subjects. Eur Respir $J$ 2000:15:590-5.

13. Bloch KE. Polysomnography: a systematic review. Technol Health Care 1997:5:285-305

14. Sackner MA, Watson $H$, Belsito AS, et al. Calibration of respiratory inductive plethysmograph during natural breathing. J App/ Physiol 1989;66:410-20.

15. Thurnheer R, Xie X, Bloch KE. Accuracy of nasal cannula pressure recordings for assessment of ventilation during sleep. Am J Respir Crit Care Med 2001;164:1914-19.

16. Maarsingh EJ, van Eykern LA, Sprikkelman AB, et al. Respiratory muscle activity measured with a noninvasive EMG technique: technical aspects and reproducibility. J Appl Physiol 2000;88:1955-61.

17. Kohler M, Kriemler S, Wilhelm EM, et al. Children at high altitude have less nocturnal periodic breathing than adults. Eur Respir J 2008;32:189-97.

18. Senn 0, Clarenbach CF, Kaplan V, et al. Monitoring carbon dioxide tension and arterial oxygen saturation by a single earlobe sensor in patients with critical illness or sleep apnea. Chest 2005;128:1291-6.

19. Rechtschaffen A, Kales A. A manual of standardized terminology, techniques and scoring system for sleep stages of human subjects. Washington, DC: Public Health Service, US Government Printing Office, 1968.

20. Sleep Disorders Atlas Task Force of the American Sleep Disorders Association. EEG arousals: scoring rules and examples. Sleep 1992;15:174-84.

21. American Academy of Sleep Medicine Task Force. Sleep-related breathing disorders in adults: recommendations for syndrome definition and measurement techniques in clinical research. Sleep 1999;22:667-89.

22. Brack T, Thuer I, Clarenbach CF, et al. Daytime Cheyne-Stokes respiration in ambulatory patients with severe congestive heart failure is associated with increased mortality. Chest 2007;132:1463-71.

23. Hoshikawa M, Uchida S, Sugo T, et al. Changes in sleep quality of athletes unde normobaric hypoxia equivalent to 2,000-m altitude: a polysomnographic study. J App/ Physiol 2007;103:2005-11.

24. Burgess KR, Johnson PL, Edwards N. Central and obstructive sleep apnoea during ascent to high altitude. Respirology 2004;9:222-9.

25. White DP, Gleeson K, Pickett CK, et al. Altitude acclimatization: influence on periodic breathing and chemoresponsiveness during sleep. J Appl Physiol 1987;63:401-12 
26. Dempsey JA, Smith CA, Przybylowski T, et al. The ventilatory responsiveness to CO(2) below eupnoea as a determinant of ventilatory stability in sleep. J Physiol 2004;560:1-11.

27. Xi L, Smith CA, Saupe KW, et al. Effects of rapid-eye-movement sleep on the apneic threshold in dogs. J Appl Physiol 1993;75:1129-39.

28. Parisi RA, Santiago TV, Edelman NH. Genioglossal and diaphragmatic EMG responses to hypoxia during sleep. Am Rev Respir Dis 1988;138:610-16.

29. Younes M. Role of respiratory control mechanisms in the pathogenesis of obstructive sleep disorders. J Appl Physiol 2008;105:1389-405.

30. Beaumont M, Batejat D, Pierard C, et al. Zaleplon and zolpidem objectively alleviate sleep disturbances in mountaineers at a 3,613 meter altitude. Sleep 2007:30:1527-33
31. Anholm JD, Powles AC, Downey R III, et al. Operation Everest II: Arterial oxygen saturation and sleep at extreme simulated altitude. Am Rev Respir Dis 1992:145:817-26.

32. Khoo MC, Anholm JD, Ko SW, et al. Dynamics of periodic breathing and arousal during sleep at extreme altitude. Respir Physiol 1996;103:33-43.

33. Somers VK, Mark AL, Abboud FM. Potentiation of sympathetic nerve responses to hypoxia in borderline hypertensive subjects. Hypertension 1988;11:608-12.

34. Ryan CM, Usui K, Floras JS, et al. Effect of continuous positive airway pressure on ventricular ectopy in heart failure patients with obstructive sleep apnoea. Thorax 2005:60:781-5.

35. Loeppky JA, Roach RC, Maes D, et al. Role of hypobaria in fluid balance response to hypoxia. High Alt Med Biol 2005;6:60-71.

\section{Lung alert}

\section{Newly identified genetic determinants of lung function}

It is well established that pulmonary function is in part genetically determined. These authors report a meta-analysis of genome-wide association study (GWAS) results from 20288 participants and follow-up analyses in 54276 participants, identifying five novel genome-wide significant loci for pulmonary function. A companion manuscript from the CHARGE Consortium, which reports a GWAS of lung function in 20890 participants, also identifies significant associations at three of these sites. These loci were not specific to smokers, genetic factors that influence smoking behaviour or adjustments for smoking exposure. The most probable candidate genes in the regions identified seem to be involved in developmental pathways important for lung growth or tissue remodelling.

While the authors admit that these determinants have been incompletely adjusted for height and were based on cross-sectional measures of lung function, these findings are important for two reasons. First, previous studies in chronic obstructive pulmonary disease (COPD) have reported changes at similar loci, thus raising the possibility that the development of COPD may be predetermined during lung development and further influenced by cigarette smoke exposure. However, it also raises the possibility that the move to define the diagnosis of COPD by abnormal lung function has led to the identification of confounding loci that are simply determinants of lung development rather than disease specific. Mechanistic and pathogenetic models are required to help determine which is the more accurate. Secondly, the identification of multiple loci involved in early lung development allows for a more targeted determination of the mechanisms by which the airways develop and respond to external insult. Detailed longitudinal studies will be required to define and address these issues.

As always with genome studies, more questions are generated than answered, but these papers provide strong evidence for newly identified genetic loci that act as important determinants of pulmonary function.

Repapi E, Sayers I, Wain LV, et al. Genome-wide association study identifies five loci associated with lung function. Nat Genet 2010;42:36-44.

Neil Martin

Correspondence to Neil Martin, Clinical Research Fellow, Institute for Lung Health, Glenfield Hospital, Leicester, UK; nmartin@doctors.org.uk

Thorax 2010;65:435. doi:10.1136/thx.2010.136267 